\title{
Apprentissage par projet en électricité : exemple et mise en oeuvre
}

\section{( titre court : Apprentissage par projet en électricité )}

\author{
L. De Vroey ${ }^{*}$, F. Vrins, F. Labrique, C. Trullemans, C. Eugène, D. Grenier \\ Département d’Electricité - Université catholique de Louvain \\ 3, Place du Levant - 1348 Louvain-la-Neuve \\ Belgique
}

\{devroey, labrique, eugene, grenier\}@ lei.ucl.ac.be

\{vrins, c.trullemans\}@ dice.ucl.ac.be

Laurent De Vroey a obtenu le diplôme d'Ingénieur Civil électromécanicien à l'Université Catholique de Louvain (UCL) en 2002 et le DEA en sciences appliquées en 2004. Il est actuellement assistant de recherche au département d'électricité de l'UCL, où il encadre les travaux pratiques de différents cours d'électricité. Ses domaines de recherche sont la modélisation et la simulation du comportement électromécanique de bio-microsystèmes.

Frédéric Vrins a obtenu le diplôme d'Ingénieur Civil électromécanicien à l'UCL en 2002 et le DEA en sciences appliquées en 2004. Il est actuellement assistant de recherche au département d'électricité de l'UCL, où il encadre les travaux pratiques de différents cours d'électricité. Il effectue une thèse de doctorat au sein du "Machine Learning Group". Ses sujets de recherche concernent notamment l'analyse en composantes indépendantes, la sélection de variables et l'entropie de mélange de variables aléatoires.

Francis Labrique est Professeur à la faculté des sciences appliquées de l'UCL. Il y enseigne les circuits électriques, l'électronique de puissance et la conversion électromécanique. Ses domaines de recherche se situent en électronique de puissance et ses applications. De 1985 à 1991 il a été professeur associé invité à l'Université technique de Lisbonne et en 1997 à l'ENS de Cachan. Il est co-auteur de neuf livres (en français, en anglais et en portugais) sur l'électronique de puissance, l'électrotechnique et l'instrumentation électronique.

Charles Trullemans est Professeur ordinaire au Laboratoire de microélectronique de l'UCL, Docteur en sciences appliquées, UCL 1974. Il est chercheur et enseigne dans le domaine de l'électronique, et plus particulièrement de la conception de systèmes intégrés mixtes. Responsable du Laboratoire de microélectronique de 1991 à 1995, Doyen de la Faculté des sciences appliquées (FSA) de l'UCL de 1995 à 2000 ; il est coordinateur des projets ESPRITLTR MIVIP et IST OPTIVIP : réalisation de prothèses implantées de stimulation neurale.

Christian Eugène est Ingénieur Civil électricien et Docteur en Sciences Appliquées de l'UCL. Professeur ordinaire au département d'Electricité de l'UCL, il y enseigne les Mesures Electriques, l'Instrumentation et les Capteurs, l'Eclairage, l'Electricité générale. Il est l'auteur ou co-auteur d'environ 120 publications dans des revues scientifiques internationales et/ou communications à des congrès scientifiques sur des recherches qui relèvent principalement du domaine de la Mesure et de l'Instrumentation. Membre actif de plusieurs associations internationales.

Damien Grenier est un ancien élève de l'Ecole Normale Supérieure de Cachan où il a obtenu le doctorat en 1994.Après un séjour post-doc à l'Ecole de Technologie Supérieure (Montréal, Canada), il a rejoint l'UCL en 1996. Depuis septembre 2005, il est professeur des universités à 
l'Antenne de Bretagne de l'ENS de Cachan. Ses domaines de recherche sont la modélisation et la commande des systèmes électromécaniques. Il est impliqué dans l'enseignement par projet de ces matières ainsi que dans le développement de ressources multimédia en ligne (projet e-lee).

*auteur à qui toute correspondance doit être adressée

\section{RESUME}

La formation d'ingénieur à l'Université catholique de Louvain a été réorganisée selon un principe de pédagogie active (apprentissage par problèmes et par projets). Ainsi, il est proposé aux étudiants qui entament leur spécialisation en électricité ou en électromécanique, un projet leur permettant d'aborder à la fois les notions d'électromagnétisme et les notions de circuits électriques et de faire le lien entre ces deux matières. Dans cet article, nous présentons en détails un de ces projets, consistant pour les étudiants à réaliser un capteur de suivi de ligne, ainsi que son électronique de traitement. Des variantes sont également exposées, s'articulant autour de différents types de capteurs intégrés. Nous détaillons les moyens et l'approche mis en œuvre et tirons les conclusions relatives à l'organisation de ces projets.

\section{Mots-clés}

apprentissage par projet, capteurs, électromagnétisme, circuits électriques, suivi de ligne

\section{INTRODUCTION}

Depuis quatre ans, la Faculté des Sciences Appliquées de l'Université Catholique de Louvain (UCL, Louvain-la-Neuve, Belgique) a effectué un virage pédagogique en adoptant une démarche d'apprentissage actif, à l'instar de ce qui se fait dans plusieurs autres universités européennes et nord-américaines [1].

Dans cette approche pédagogique, les étudiants sont confrontés à des problèmes ou des projets concrets qui les incitent à rechercher par eux-mêmes les connaissances dont ils ont besoin pour les résoudre, plutôt que d'attendre plus ou moins passivement que l'enseignant les leur apporte. Les canaux traditionnels de transmission des savoirs, les cours, les livres et les notes de référence ont été évidemment maintenus et constituent même souvent la source principale où puisent les étudiants.

Les problèmes et les projets peuvent en première approche être vus comme une source de motivation supplémentaire pour assister aux cours ou ouvrir les livres. Les projets permettent en plus la confrontation à la réalité expérimentale. Ils sont également une excellente opportunité pour une approche interdisciplinaire des problèmes d'ingénierie. Les étudiants y travaillent en groupe, tantôt entre eux, tantôt avec l'aide d'un tuteur ou d'une autre personne ressource. Ils sont invités à proposer des solutions, les comparer, les commenter et les valider que ce soit par simulation numérique ou au travers d'une réalisation concrète, bref à se former au véritable métier d’ingénieur. 
Des cours de restructuration permettent ensuite de synthétiser les nombreuses notions approchées par les différents groupes, de voir dans quelle mesure certaines conclusions peuvent ou non être généralisées, d'ouvrir vers des sujets voisins où les mêmes approches, les mêmes méthodologies pourraient éventuellement s’appliquer.

Le travail en groupe, au-delà de l'apprentissage technique proprement dit, est également une excellente opportunité pour les étudiants d'aborder les aspects humains du travail d'ingénieur, puisqu'ils sont amenés à organiser efficacement le travail, échanger leurs expériences, émettre des idées et des avis sur les idées des autres, pour finalement choisir, réaliser et défendre ensemble leur solution.

Les projets d'électricité présentés ci-dessous s’adressent à des étudiants ingénieurs civils [2], qui entament une spécialisation en électricité ou en électromécanique. Le retour d'expérience décrit ici concerne des étudiants évoluant au sein de l'ancien système (2 ans de tronc commun + 3 années de spécialité). Il est donc relatif à un projet se déroulant au cours du premier semestre de la $3^{\mathrm{e} m e}$ année. Le nouveau système issu de la réforme de Bologne prévoit un premier cycle de 3 ans (dit baccalauréat en Belgique) avec une pré-spécialisation au bout d'un an et demi, suivi d'un second cycle de 2 ans (dit maîtrise). Il est prévu que le projet présenté ici (ou une évolution de celui-ci) soit alors donné au second semestre de la deuxième année.

Dans la suite de cet article, nous présentons d'abord le canevas des projets proposés aux étudiants, en détaillant leurs objectifs et la mise en œuvre pratique de ceux-ci.

Ensuite, nous exposons de manière détaillée l'un de ces projets, en insistant sur la manière dont se sont déroulées les interactions entre étudiants et équipe enseignante.

Nous proposons alors certaines variantes de ce projet et insistons sur les points communs et différences d'approche entre celles-ci et le premier projet présenté.

Enfin, nous tirons les conclusions de notre expérience des projets dans le cadre de la formation en électricité des étudiants ingénieurs.

\section{OBJECTIFS}

L’énoncé du projet est donné tout au début de la spécialisation en électricité ou électromécanique. A ce stade de leurs études, les étudiants ont déjà une connaissance de base des circuits électriques : lois topologiques, relations constitutives des éléments, équations d'évolution de circuits du premier ordre, notions élémentaires sur les circuits soumis à des courants et tensions sinusoïdaux. Ils ont de même abordé les équations de Maxwell dans le vide.

Au cours du semestre, ces notions seront revues et complétées. Seront notamment abordés l'analyse fréquentielle des circuits électriques (transformation de Laplace, diagramme de Bode), les opérations simples de traitement de signal et l'amplificateur opérationnel idéal. On y insistera surtout sur la mise en application des ces notions théoriques, que ce soit dans les cours où des dispositifs particuliers seront décrits et analysés ou dans le cadre du projet où l'on demandera en outre aux étudiants de concevoir un circuit électronique capable d'effectuer la mesure d'une grandeur physique. 
Le projet, qui représente un volume de 5 crédits (ECTS), interagit étroitement avec les cours de "Circuits et mesures électriques - 7 crédits » et d' " Electromagnétisme -5 crédits ». Il constitue également pour tout ce qui concerne l'analyse fréquentielle, une mise en pratique du cours de "Mathématiques appliquées : signaux et systèmes - 5 crédits ». Contextualisant ainsi 22 crédits sur les 30 que compte le semestre, le projet représente un poids important dans ce début de formation en électricité et en électromécanique. En schématisant, on peut dès lors dire que son objectif pédagogique coïncide quasiment avec les objectifs généraux du semestre en question.

Dans le dispositif pédagogique mis en place, le rôle spécifique du projet est :

- de faire le lien entre la théorie des circuits et l'électromagnétisme ;

- d'aider l'étudiant à se familiariser avec les opérations simples de traitement de signal (amplification, sommation, filtrage) ;

- de l'initier à la conception de circuits électriques en développant ses capacités d'analyse des circuits, en le poussant à décomposer ceux-ci en sous-circuits ayant chacun une fonction spécifique, en lui faisant dimensionner les composants ;

- de lui enseigner une utilisation critique des outils de simulation en vue de la validation d'un schéma (construction a priori d'une idée de la réponse d'un circuit, comparaison avec le résultat de simulation, comparaison de ce résultat avec le résultat de mesure) ;

- enfin, de développer en lui des capacités d'expérimentateur : réalisation de montages électriques et électroniques simples, utilisation de l'appareillage courant de laboratoire (sources, générateurs de signaux, oscilloscopes, multimètres), identification des causes d'écart de comportement entre un circuit réel et son modèle, corrections éventuelles, examen critique des erreurs de mesure, compte tenu des performances des appareils.

\section{MISE EN OEUVRE}

\subsection{PLANNING}

Les étudiants travaillent par groupes de 4, librement constitués. Habituellement, une quinzaine de groupes sont ainsi formés. Ils disposent d'une dizaine de semaines en moyenne pour mener à bien le projet proposé (figure 1).

Le mode d'organisation des étudiants au sein du groupe est laissé libre également. Ces étudiants sortent en effet de deux années de formation méthodologique au travail en groupe [1] et sont jugés suffisamment mûrs pour s'organiser par eux-mêmes, que ce soit en reprenant les modèles qui leur ont été enseignés ou au contraire en expérimentant d'autres variantes.

Deux rencontres obligatoires avec l'équipe enseignante jalonnent le travail. Entre ces rencontres, une plage de deux heures est prévue chaque semaine par l'équipe pour répondre aux questions des étudiants et suivre l'évolution de leurs travaux. Certains étudiants exploitent beaucoup cette possibilité, d'autres n'en éprouvent pas le besoin et ne viennent même pas. 
Après trois semaines de travail, les étudiants présentent oralement devant l'équipe un rapport d'avant-projet comprenant leurs choix de solution ainsi que les problèmes rencontrés jusqu'alors. Cela oblige tout d'abord l'ensemble des groupes à faire le point sur leur avancement, à produire des résultats et à les synthétiser. C'est aussi l'occasion pour l'équipe enseignante de les encourager dans la direction choisie ou de les faire réfléchir aux problèmes inhérents à leurs choix et qu'ils n'auraient pas envisagés. C'est enfin une opportunité pour stimuler les groupes à la traîne.

Ce n'est qu'après cette première évaluation que les portes du laboratoire sont ouvertes aux étudiants. Ceci évite qu'ils passent prématurément un temps considérable à expérimenter des solutions pas ou peu réfléchies.

Une deuxième évaluation a lieu après neuf semaines de travail. Lors de cette rencontre, les étudiants présentent les résultats théoriques et pratiques de leur projet, qui doit être fonctionnel. Ils commentent le rapport qu'ils ont remis quelques jours plus tôt à l'équipe enseignante. Ils comparent leur solution finale avec leur solution d'avant-projet et tirent les conclusions qui s'imposent. Si la présentation est convaincante, celle-ci marque la fin du projet. Si la présentation de certains groupes n'est pas satisfaisante, l'équipe enseignante leur demande alors d'approfondir ou de corriger certains points d'ordre théorique qu'elle estime n’avoir pas été suffisamment étudiés ou assimilés par les groupes concernés. Ceux-ci sont alors invités à remettre, deux semaines plus tard, un rapport d'étude complémentaire, qui permettra à l'équipe enseignante de rendre un avis final éventuellement plus favorable sur leur travail. Cette démarche est donc une chance laissée aux étudiants d'améliorer leur compréhension et d'atteindre les objectifs d'apprentissage du projet.

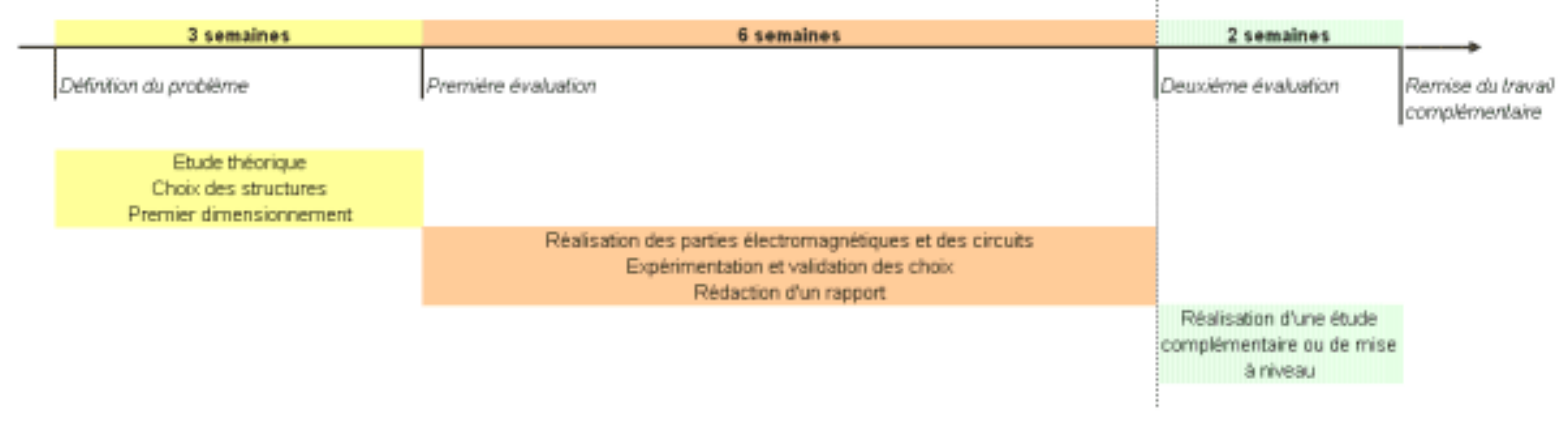

Fig. 1 - Organisation temporelle des projets

L'évaluation finale du travail des groupes par l'équipe enseignante se fait sur base des objectifs énoncés au début de celui-ci. Sauf exception, l'évaluation est globale pour les différents membres du groupe, chacun des membres étant supposé avoir acquis au même degré les différentes notions reprises dans les objectifs d'apprentissage et avoir contribué de manière équitable au résultat final. Lors de l'évaluation, il est demandé que chacun des membres du groupe s'exprime, afin de responsabiliser l'ensemble des étudiants du groupe.

\subsection{ENCADREMENT}

L'équipe pédagogique est constituée de trois types de personnes. Trois professeurs assurent la coordination et la responsabilité du projet. Ils en fixent le sujet et les objectifs et participent aux différentes rencontres avec les étudiants. Trois assistants sont dévolus à 
l'accompagnement sur le terrain des étudiants et veillent au bon avancement du projet. Ils sont les principales personnes de contact pour les étudiants en dehors des plages prévues à cet effet. Enfin, des "étudiants-moniteurs », sélectionnés parmi les étudiants de dernière année, renforcent l'encadrement pédagogique, principalement pour le volet expérimental en laboratoire. Ces étudiants ont eux-mêmes réalisé le projet deux ans auparavant et connaissent donc les difficultés rencontrées par leurs jeunes collègues. Les étudiants-moniteurs peuvent valoriser leur activité d'encadrement et les compétences pédagogiques qu'elle leur apporte, en la faisant compter pour un cours à option dans le cadre de leur cursus.

\subsection{OUTILS}

Un certain nombre d'outils de simulation sont à la disposition des étudiants (logiciels PSpice et MatLab) ; la consultation de livres de référence et de certains sites internet leur est conseillée par l'équipe enseignante, tels que le site http://www.e-lee.net, traitant de circuits linéaires.

Après la première rencontre, qui clôture l'avant-projet, les étudiants ont accès au laboratoire d'électronique pour les essais et mesures pratiques. Comme le temps d'accès leur est limité durant le projet (de 2 à 4 heures par semaine pour chaque groupe), il leur est vivement conseillé de s'organiser efficacement. Pour ce faire, il est demandé que les visites au laboratoire soient préparées, que seuls deux étudiants de chaque groupe soient présents simultanément au laboratoire, et que ces paires d'étudiants varient au cours du projet, afin que chacun s’implique dans tous les aspects du projet, et pour forcer les étudiants à partager leurs connaissances.

Au laboratoire, durant les plages horaires prévues, des membres de l'équipe enseignante sont présents. Leur rôle est d'encourager les étudiants à travailler de manière autonome : les inviter à rechercher les informations qui leur manquent dans les fiches techniques des matériels et composants, les faire réfléchir, leur expliquer comment trouver leurs erreurs,... En fin de projet, le laboratoire est également accessible aux étudiants, sans encadrement, par exemple en soirée ou le week-end.

\section{UN EXEMPLE D'APPRENTISSAGE PAR PROJET : REALISATION D'UN SYSTEME DE FILOGUIDAGE ELECTROMAGNETIQUE}

Nous présentons ci-dessous l'énoncé et l'approche suivie pour un exemple de projet réalisé à l’UCL [3] et synthétisés à la figure 2. Un cahier des charges du projet peut être consulté ici. 


\section{Les objectifs}

- faire le lien entre l'électromagnétisme et la théorie des circuits

- maittriser les opérations simples de traitement de signal

- utiliser la théorie des circuits pour concevoir, modéliser, dimensionner un système

- percevoir les limites de l'analyse par simulation et la nécessité d'une modélisation soignée

\section{Le contexte}

un système de filoguidage

suivre un fil de guidage en détectant le champ produit par un courant MF circulant dans le fil

\section{Le sujet \\ un capteur de position pour ce système \\ réaliser un capteur qui fournit une tension v \\ proportionnelle à l'écart de position par rapport au fil \\ (pour un petit écart) \\ à partir des tensions induites par le champ dans des \\ bobines sans fer \\ - en traitant de manière appropriée ces tensions}

Fig. 2 - Objectifs, contexte et sujet d'un projet particulier

\subsection{ENONCE DU PROJET}

L’énoncé présenté aux étudiants est le suivant :

Concevoir un capteur et une électronique de traitement permettant à un véhicule d'être guidé - sans contact - par un fil électrique parcouru par un courant alternatif. On demande qu'à la sortie du circuit, la fonction de transfert $V_{\text {out }} / D x$ (voir figure 3) soit linéaire autour de zéro et que son signe permette d'identifier le sens de déplacement par rapport au fil (vers la droite ou vers la gauche).
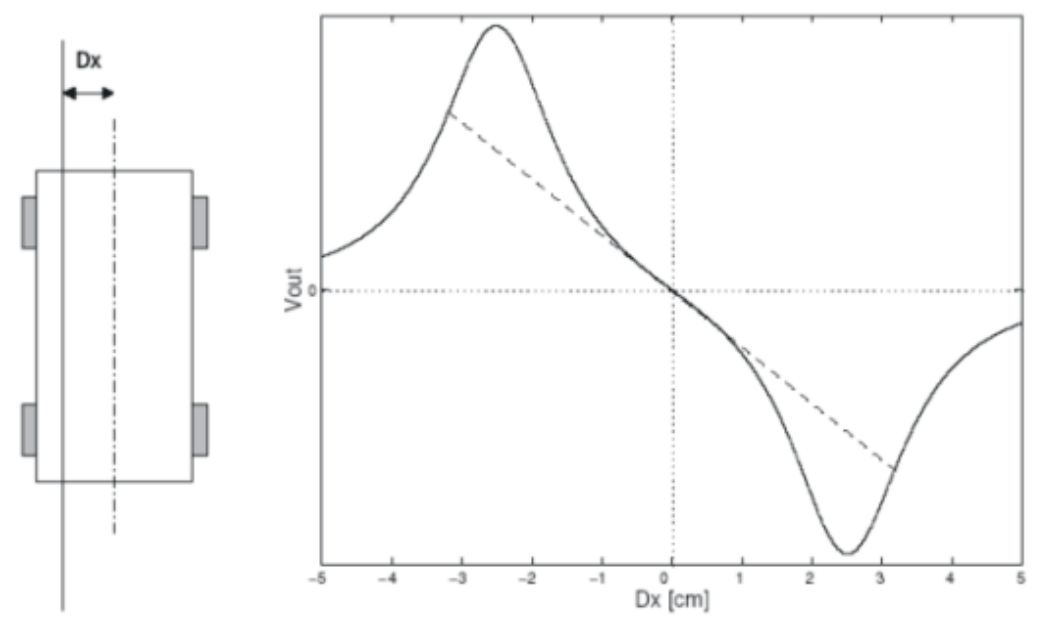

Fig. 3 - gauche : schéma du système (trait continu : fil de guidage ; trait interrompu : axe du véhicule) ; droite : allure d'une fonction Vout $=\mathrm{f}(\mathrm{Dx})$ acceptable 


\subsection{APPROCHE DES ASPECTS D’ELECTROMAGNETISME}

Le capteur choisi pour la détection de la ligne à suivre repose sur les principes de l'électromagnétisme. Avant de traiter le signal reçu par le capteur, il importe de comprendre la nature de ce signal ainsi que les éléments dont il dépend, afin de dimensionner convenablement le capteur, élément le plus critique de la chaîne de mesure. Nous décrivons dans la suite de cette section la démarche suivie pour ce faire par les étudiants.

\subsubsection{Considérations générales}

Dans ce projet, les tensions induites dans des bobines de mesure par un fil parcouru par un courant alternatif $\left(\mathrm{i}_{\text {in }}\right)$ sont exploitées pour en déduire la position du capteur par rapport au fil.

Un choix de départ de l'équipe enseignante est de ne pas faire usage d'un noyau ferromagnétique pour supporter les bobines. De cette manière, grâce à la linéarité du dispositif et à l'homogénéité magnétique du milieu, on peut plus facilement obtenir un résultat analytique pour les signaux mesurés, même si ceux-ci sont faibles vu la non-canalisation du flux.

Si l'approche se doit d'être globale, il est néanmoins nécessaire de s’intéresser aux aspects électromagnétiques de manière séparée des aspects électroniques liés au traitement des mesures, afin de percevoir l'influence des différents paramètres dimensionnels et de proposer une architecture adéquate pour les bobines.

Comprendre l'influence de la position, du nombre de spires, du diamètre,... des bobines sur la tension induite aux bornes de celles-ci est primordial et représente pour les étudiants la première approche pratique de l'électromagnétisme dans leur cursus universitaire.

\subsubsection{Etude de la tension induite dans une bobine}

Initialement, aucune indication n'est donnée concernant le nombre, les dimensions, la position ou les caractéristiques techniques des bobines nécessaires à la réalisation d'un capteur de suivi de ligne.

Une première étude est suggérée aux étudiants, qui est de déterminer la tension aux bornes d’une bobine soit horizontale, soit verticale, comme résumé à la figure 4.

Des résultats semblables, pour différents jeux de données, peuvent être obtenus au moyen d'un programme matlab. Dans ce programme, nous présentons un calcul des tensions induites dans des bobines de section carrée pour différentes configurations. On y compare aussi les résultats obtenus si l'on tient compte de la hauteur et de l'épaisseur des bobines ou si on considère que toutes les spires sont concentrées. 


\section{Les bobines de détection}

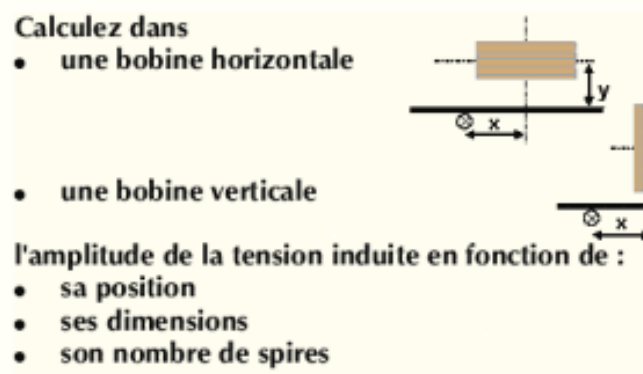

l'amplitude de la tension induite en fonction de :

- sa position

- ses dimensions

- son nombre de spires
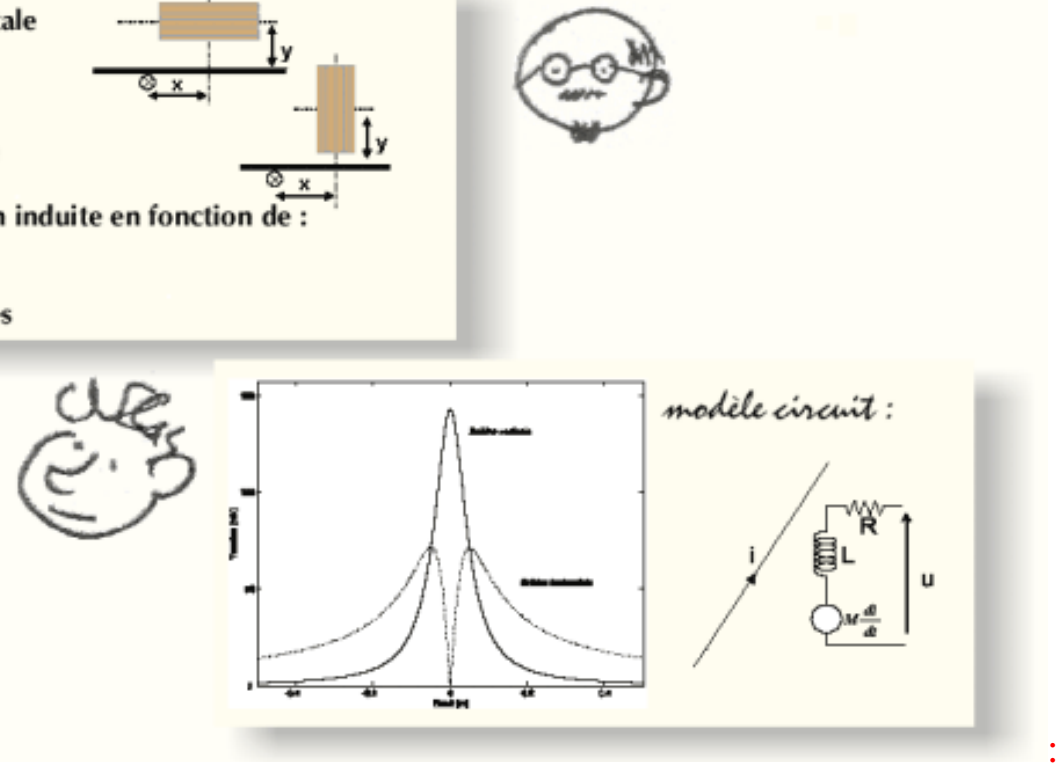

Fig. 4 - Synthèse de la démarche

A ce stade, les étudiants considèrent les seuls aspects d'inductance mutuelle entre le fil de guidage et les bobines en calculant la force électromotrice induite par le courant parcourant le fil.

Se basant sur les lois de l'électromagnétisme (Lenz, Faraday, Ampère), les étudiants s'intéressent analytiquement aux effets du nombre de spires, du diamètre, de la longueur, des distances (horizontale $\mathrm{x}$ et verticale y) des bobines par rapport au fil véhiculant le courant, sur la tension induite aux bornes des bobines. Ce faisant, ils prennent conscience qu'il est possible d'utiliser des modèles simplifiés, à condition d'en percevoir les limites.

\subsubsection{Un capteur, deux bobines}

Sur base de leur étude précédente, les étudiants découvrent assez vite que pour obtenir une information complète sur l'amplitude et le signe de l'écart au fil, le recours à deux bobines est nécessaire. Les recherches personnelles des étudiants, la concertation entre les groupes ainsi que les pistes suggérées par l'équipe enseignante les conduisent rapidement vers différentes configurations géométriques.

Une première configuration, que nous désignerons par conf_a dans la suite, consiste à placer les bobines sur un même axe horizontal, transversal à la direction du fil (figure 5). 


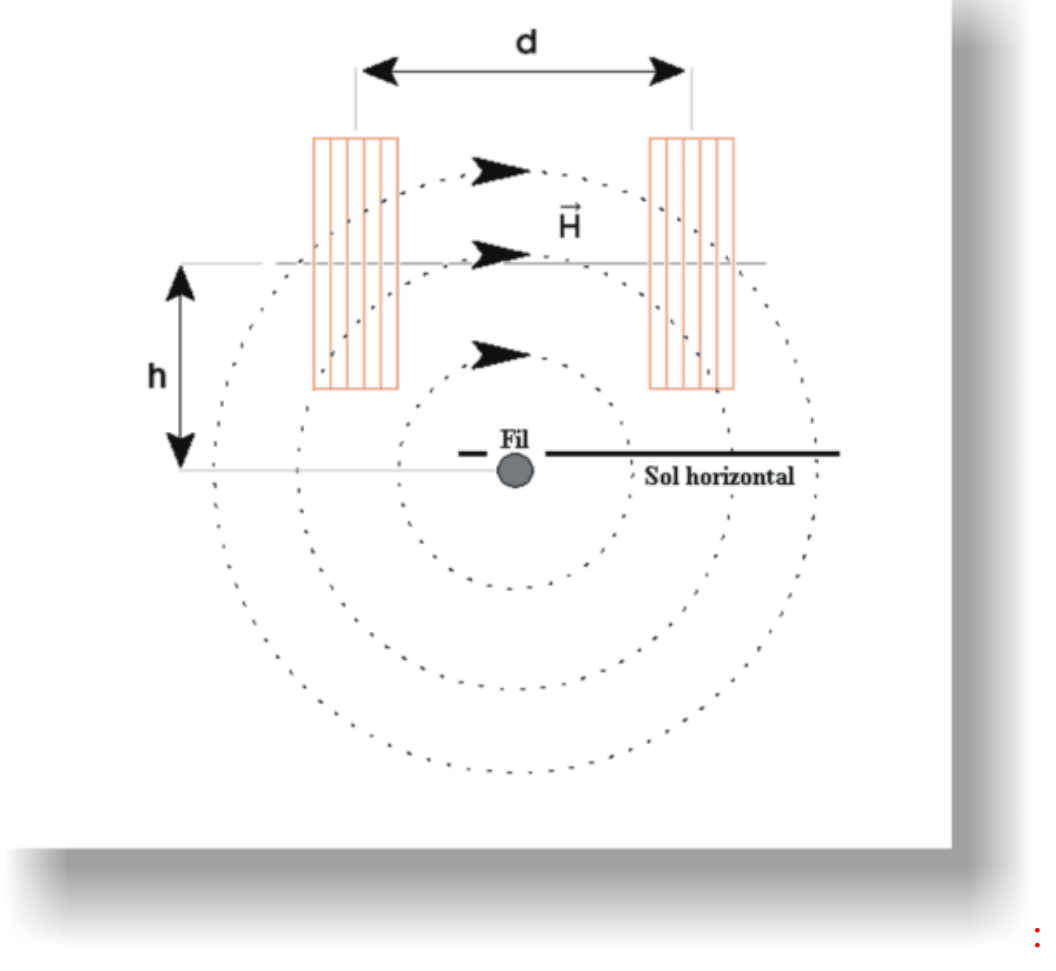

Fig. 5 - Première configuration des bobines (conf_a)

Une deuxième configuration, conf_b, assez proche de la première, consiste à placer les bobines à la même position que dans le premier cas, mais avec leur axe vertical (figure 6).

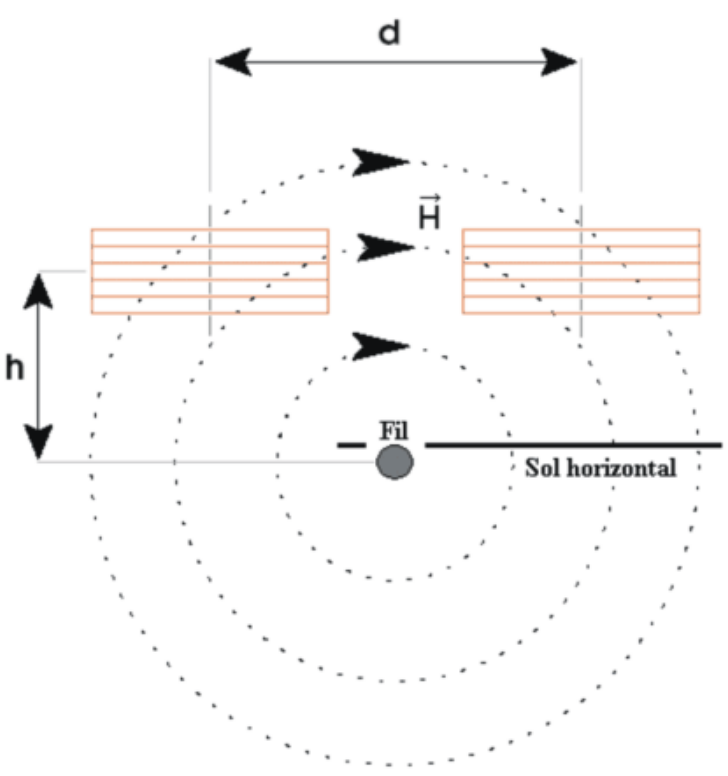

Fig. 6 - Deuxième configuration des bobines (conf_b) 
Une troisième configuration, conf_c, conceptuellement différente des deux premières, consiste à orienter l'axe d'une des bobines horizontalement et transversalement à la direction du fil, et l'axe de la seconde verticalement et en-dessous de la précédente (figure 7).

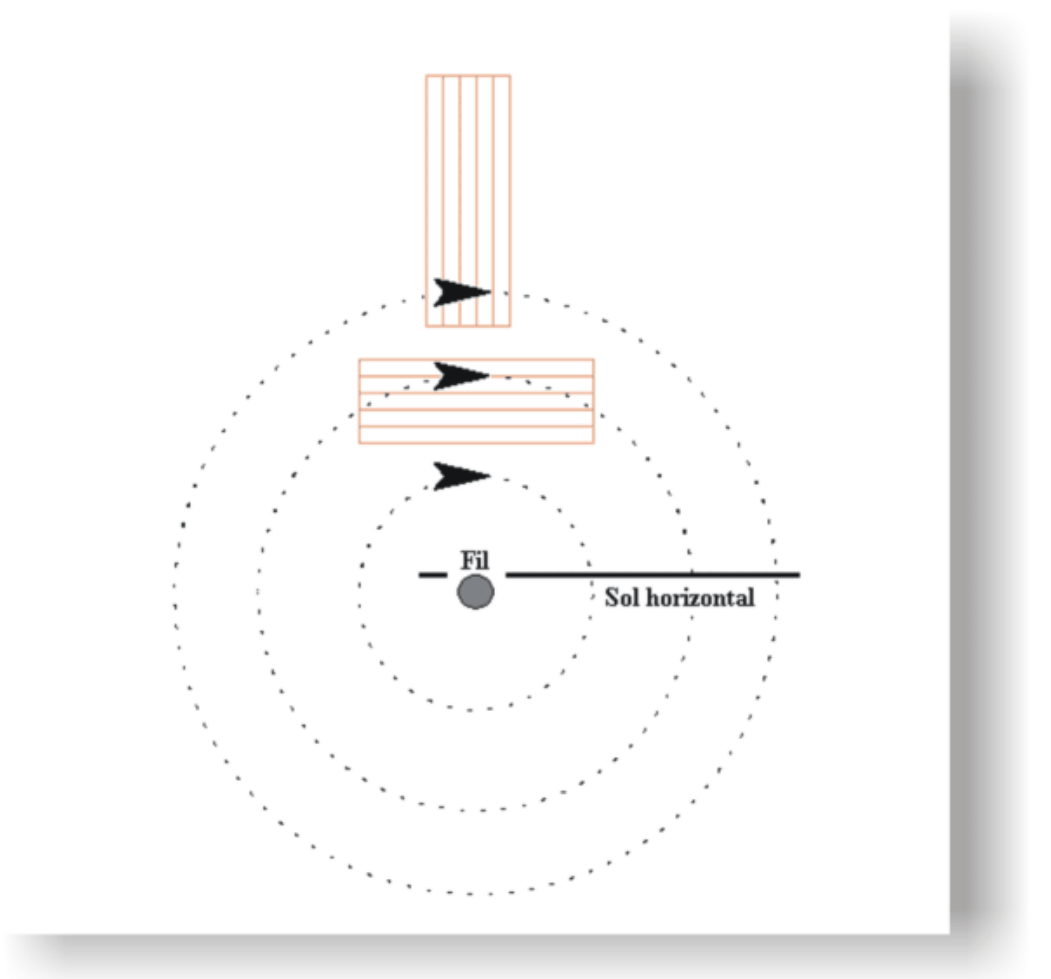

Fig. 7 - Troisième configuration des bobines $($ conf_c)

L'allure de la différence d'amplitude entre les tensions mesurées par chaque bobine pour les deux premières configurations est présentée ci-dessous (figure 8).

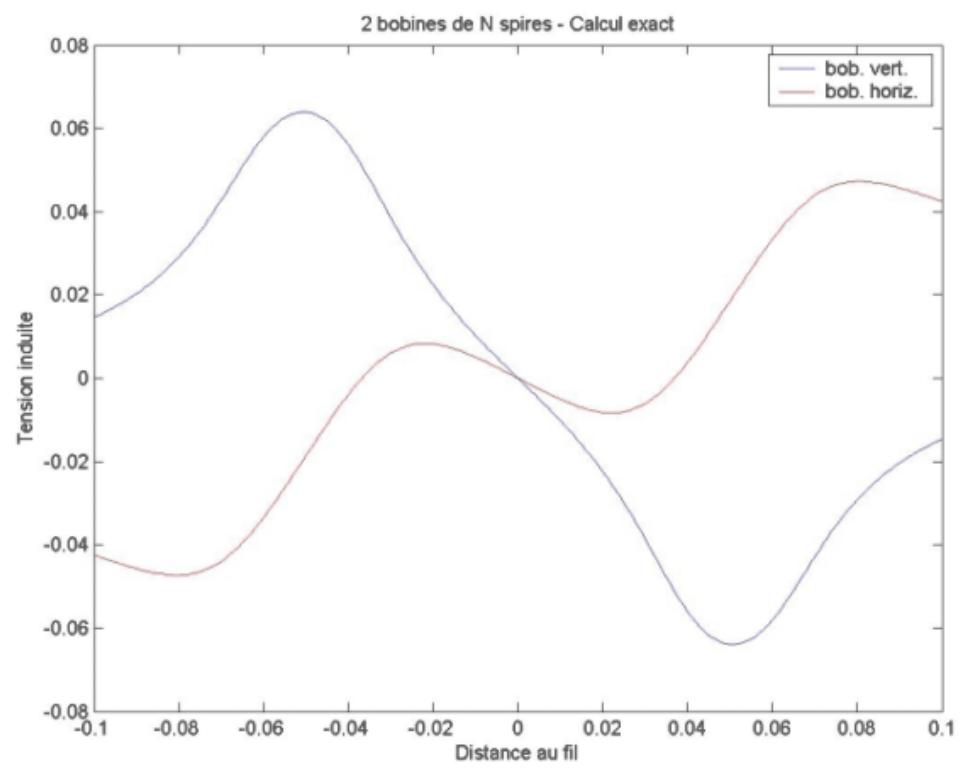

Fig. 8 - Comparaison des différences entre les amplitudes des tensions induites dans chaque bobine pour les configurations conf_a (en bleu) et conf_b (en rouge) 
Dans ces deux cas, il existe une plage de variation linéaire de la tension à proximité du fil. Néanmoins, la réponse de conf_a présente une meilleure sensibilité. La solution conf_b est donc rejetée par les étudiants soucieux de la qualité finale de leur chaîne de mesure.

Dans le troisième cas, les deux bobines jouent des rôles différents. L’amplitude de la tension dans la bobine d'axe vertical détermine la distance au fil. A même distance, à gauche et à droite du fil, les amplitudes sont les mêmes mais la phase diffère de $180^{\circ}$. Le signal de la bobine d'axe horizontal présente une phase constante, quel que soit le côté duquel se trouve le capteur. Dès lors, ce signal peut être utilisé comme élément de synchronisation.

A ce stade, les étudiants sont confrontés à deux difficultés. La première difficulté réside dans la non unicité de la solution ; la confrontation au choix et à ses justifications est nouvelle pour eux et pas toujours facile à gérer. La seconde difficulté réside dans le fait qu'un certain nombre de ces choix dépend directement des caractéristiques du circuit de traitement qui devra suivre: Quelles tensions doit-on fournir au circuit? Quels courants le circuit prélèvera-t-il sur les bobines? C'est ici qu'apparaît la charnière entre le problème électromagnétique et le problème électronique, qui fait prendre conscience aux étudiants que la réalisation d'une chaîne de mesure nécessite une prise en compte globale de ces différents éléments.

La plupart des groupes ont opté pour la première configuration, simple dans la compréhension. Certains groupes, encouragés par l'équipe enseignante, se sont toutefois concentrés sur la troisième configuration, dont ils ont pu apprécier les avantages lors de la réalisation de la partie électronique (figure 9). Le développement de la chaîne de traitement pour ces deux configurations est détaillé dans la section IV.3.

L'amplitude de la tension dans une bobine dépend de $|\mathrm{Dx}|$. Comment savoir si on est à gauche ou à droite du fil ?
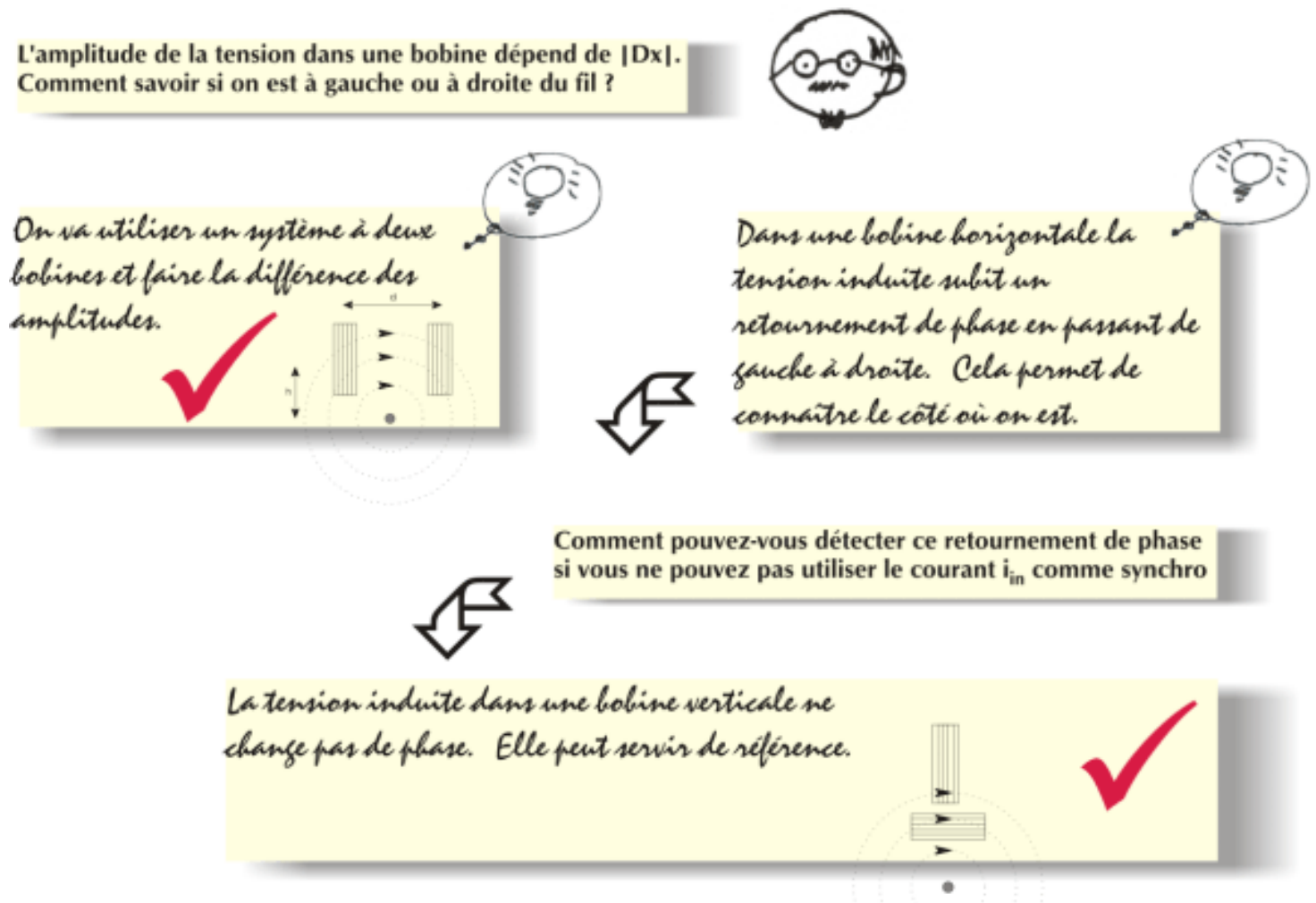

Fig. 9 - Synthèse de la démarche 


\subsubsection{Réalisation et test des bobines}

La réalisation des bobines et les premières mesures sur celles-ci révèlent aux étudiants toute la complexité du problème. S'ils se doutaient qu'un fil de cuivre n’avait pas une résistivité nulle, et que le fait de bobiner un nombre relativement important de spires devait assez logiquement engendrer une inductance non négligeable, ils réalisent à ce moment que la tension théoriquement induite par le courant circulant dans le fil de guidage et la tension effectivement mesurée aux bornes de la bobine pouvaient être relativement différentes. Pire : le rapport entre ces tensions varie avec la fréquence d'alimentation et présente même un maximum !

Une fois remis de leurs émotions, crayon à la main, ils s'efforcent de proposer une explication rationnelle à ces phénomènes : la bobine est en réalité un élément partiellement résistif, inductif et capacitif (figure 10). Le tout est maintenant de représenter schématiquement ces éléments distribués, d'une façon qui corresponde au mieux au sens physique, et de les dimensionner. La capacité, provenant des phénomènes diélectriques entre spires, apparaît en parallèle avec le modèle adopté initialement pour la bobine. Son effet est de créer un diviseur d'impédance avec les composantes résistive et inductive, ce qui affecte la valeur de la tension mesurée, rendant celle-ci différente de la tension induite, avec un rapport qui dépend de la fréquence.

L'étude fréquentielle du comportement des bobines et les diagrammes de Bode qu'ils réalisent sur base des éléments calculés leur permettent de réconcilier théorie et pratique, en adoptant un modèle plus réaliste.

Relevez expérimentalement la tension induite en faisant varier la fréquence du courant $i_{\text {in }}$
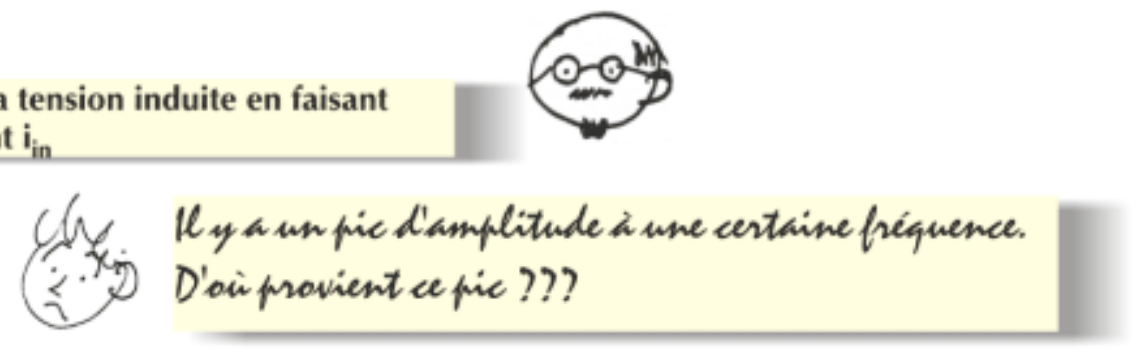

Mesurez au pont de Maxwell les paramètres de la bobine pour différentes fréquences

$$
\text { Uy aune capacité "parasite". D'oì provient-elle? }
$$

C'est la capacité interspires.

Elle était prévisible, mais difficilement calculable. Cette capacité vient en parallèle avec votre premier modèle.

Fig. 10 - Synthèse de la démarche

Les étudiants se rendent compte que pour modéliser l'impédance à partir des mesures, ils doivent travailler à plusieurs fréquences. Les mesures au pont d'impédance, ainsi que les notions de fréquence de résonance, les aident alors à caractériser les aspects électriques des bobines. Une idée leur vient: Pourquoi ne pas travailler à la fréquence de résonance des bobines ? De cette manière, nous aurons en entrée du circuit électronique une tension de 
grande amplitude qui sera plus facile à traiter! Les groupes qui suivent cette piste tête baissée et sans autre considération, certains de leur fait, se brûlent les ailes en se rendant compte que si la résistance et l'inductance sont en principe assez proches pour deux bobines semblables, il n'en est pas de même de la capacité parasite, qui dépend fortement de la manière dont ont été réalisés les bobinages. De ce fait, les fréquences de résonance des deux bobines peuvent être différentes. Si les étudiants règlent la fréquence du courant dans le fil inducteur sur la fréquence de résonance de l'une des bobines, la réponse de l'autre bobine risque d'être fort différente en amplitude, ce qui est gênant dans les deux premières géométries présentées, ou en phase, ce qui est gênant dans la troisième.

Une solution suggérée par l'équipe enseignante est de placer en parallèle avec chacune des deux bobines une capacité de valeur nettement plus élevée que la capacité parasite, afin d'imposer aux deux montages une capacité approximativement égale et donc une fréquence de résonance commune aux deux bobines (figure 11).
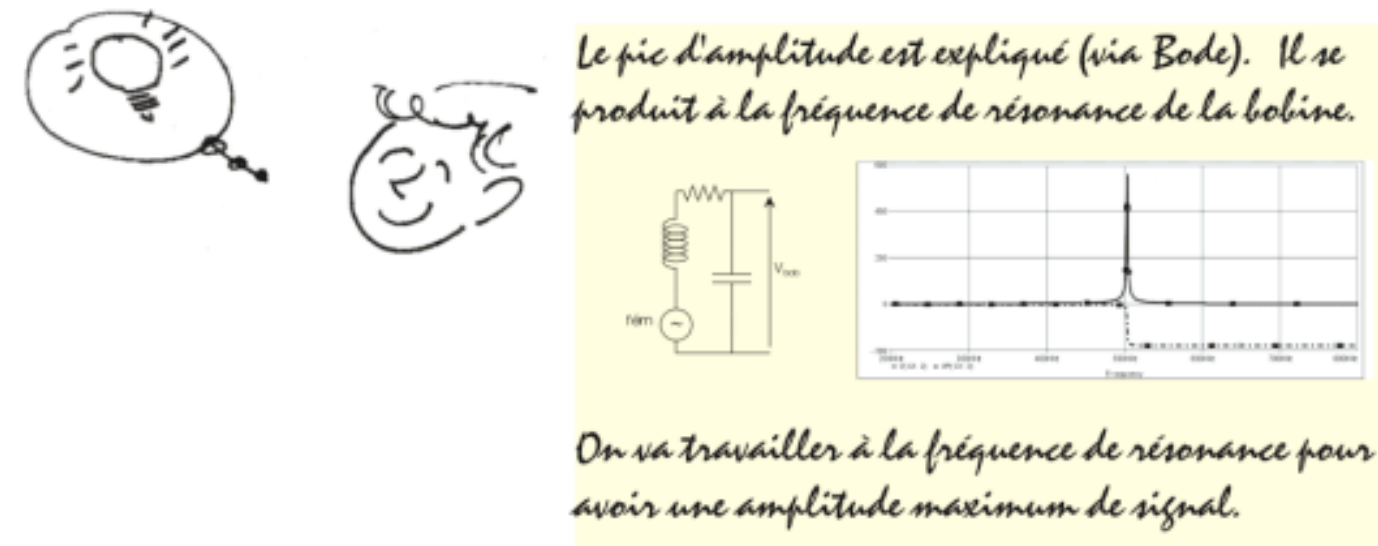

La capacité interspires dépend fort des conditions de fabrication.

Elle peut varier d'une bobine à l'autre pour des bobines théoriquement identiques.
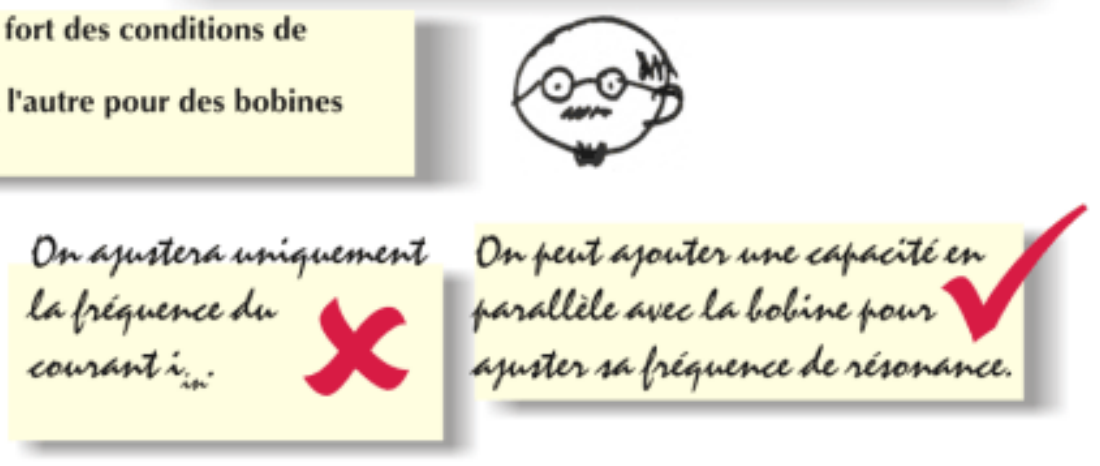

Fig. 11 - Synthèse de la démarche

\subsubsection{Observations}

Grâce à la réalisation pratique des bobines, les étudiants ont pu approcher concrètement les lois de l'électromagnétisme. Ils ont pu se rendre compte des ordres de grandeur nécessaires pour les différents paramètres ainsi que des effets non négligeables d'un désappariement des bobines. Ils ont pu faire le lien entre l'électromagnétisme (par le biais de la force électromotrice induite) et l'électronique (par le biais de la tension totale mesurée aux bornes de la bobine). 


\subsection{APPROCHE DES ASPECTS ELECTRONIQUES LIES AU TRAITEMENT DES MESURES}

Le rôle des bobines présentées dans la section 4.2. est de transformer un champ magnétique variable, résultant du passage d'un courant alternatif dans un fil, en une tension alternative. Une étude approfondie de ces « convertisseurs » a permis d'exprimer la fonction de transfert liant le courant dans le fil (signal d'entrée $\mathrm{i}_{\text {in }}$ ) en une tension alternative, mesurée aux bornes des bobines $\left(\mathrm{v}_{\mathrm{b}}\right)$. Toutefois, il reste à mettre en forme cette tension alternative. Conformément aux énoncés, il était demandé de fournir une tension continue $\mathrm{v}_{0}$. Un traitement de signal analogique assure cette conversion. L'étude électromagnétique nous donne la fonction de transfert entre $i_{i n}$ et $v_{b}$, alors que la chaîne de traitement de signal engendrera une fonction de transfert entre $\mathrm{v}_{\mathrm{b}}$ et $\mathrm{v}_{\mathrm{o}}$.

\subsubsection{Considérations générales}

Les étudiants ont été invités à proposer une chaîne de traitement de signal sous forme de blocs fonctionnels. Il est utile pour cela qu'ils connaissent auparavant les montages électroniques de base (amplification, sommation, filtrage, redressement).

Le fonctionnement de l'amplificateur opérationnel est présenté à l'aide de différents modèles dans le cours d'électronique donné en parallèle. L'équipe enseignante propose également l'étude de schémas simples construits autour de cet amplificateur, qui permettent d'illustrer les concepts de contre-réaction et d'impédance d'entrée et sortie. Ces schémas de base, ou d'autres trouvés dans la littérature ou sur internet par les étudiants, sont les blocs fonctionnels avec lesquels les étudiants sont invités à construire une chaîne d'opérations mathématiques constituant le traitement du signal. Pour les aider tant dans la compréhension des schémas de base que dans la validation de leurs choix, un outil de simulation, le logiciel Pspice, est à leur disposition.

Après l'avoir présentée à l'équipe enseignante, les étudiants sont invités à réaliser et tester leur solution en laboratoire, à se rendre compte des éventuelles limites et imperfections de celle-ci, et à y remédier le cas échéant.

\subsubsection{Modèles de l'amplificateur opérationnel et blocs fonctionnels}

Durant tout le projet, les étudiants sont amenés à simuler leurs montages. Plusieurs modèles d'amplificateurs ont été proposés, du plus simple au plus compliqué.

Le modèle le plus simple de l'amplificateur opérationnel est la source de tension commandée en tension avec impédance d'entrée infinie (fichier Pspice - student version release 9.1).

La tension de sortie de l'amplificateur est proportionnelle à la tension entre les bornes d'entrée de celui-ci :

$$
v_{\text {out }}=g v_{\varepsilon} \text { avec } v_{\varepsilon} \equiv v_{+}-v_{-}
$$

Ce modèle très simple est celui qui a été donné au cours comme premier modèle de l'amplificateur opérationnel. Le gain $g$ est positif, de l'ordre de $10^{5}$, et n'est pas connu précisément. Outre le fait que la tension de sortie peut excéder les tensions d'alimentation, il souffre d'un défaut très perturbateur pour les étudiants: il donne quasiment les mêmes résultats pour des montages à contre-réaction positive et négative. Il en résulte par exemple 
qu'un montage de type Trigger de Schmidt aura en simulation la même réponse qu'un montage inverseur (figure 12).

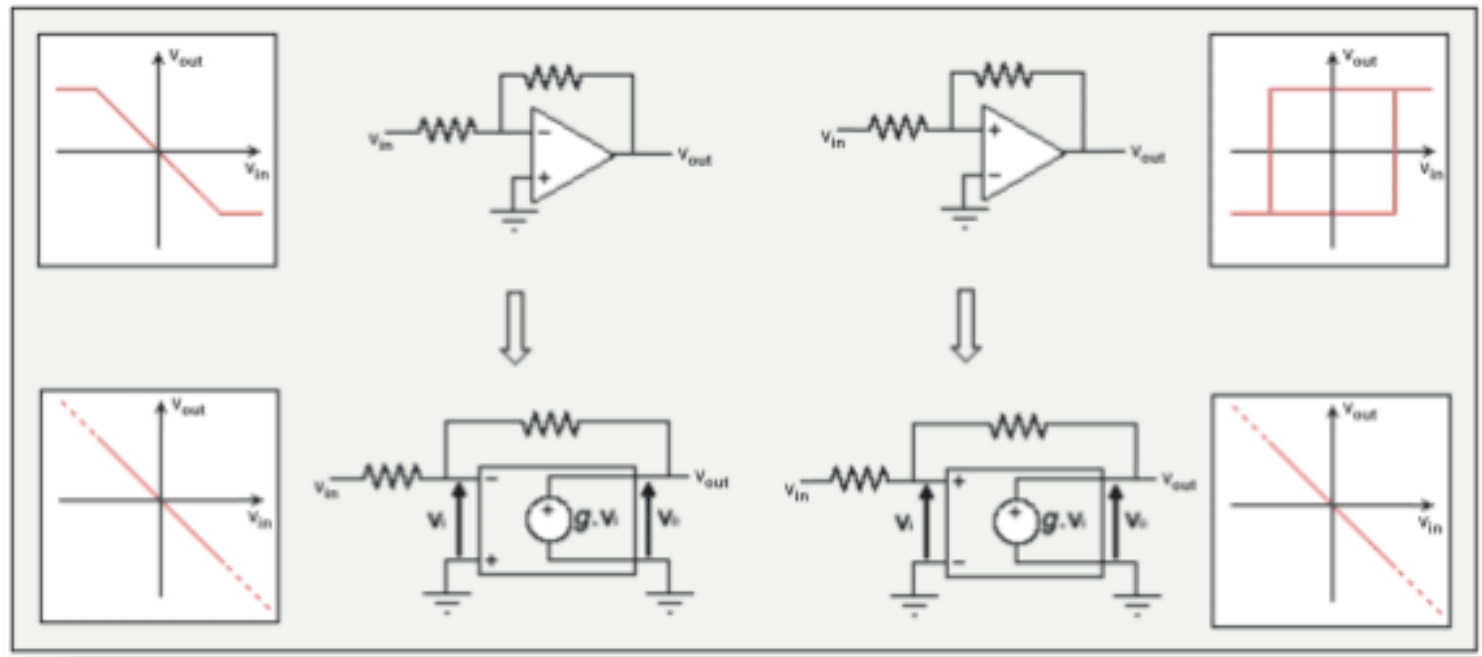

Fig. 12 - Comparaison des réponses en tension $V_{\text {out }}$ en fonction de $V_{\text {in }}$ pour le modèle simple dans deux cas de figure particuliers

La raison en est que ce modèle de l'amplificateur opérationnel est purement statique. Pour pouvoir tenir compte du comportement dynamique de l'amplificateur, et en particulier de la stabilité du montage dans lequel il s'insère, il faut ajouter un pôle à ce modèle (fichier Pspice). Les étudiants observent alors que la réaction négative stabilise l'amplificateur opérationnel, tandis que la réaction positive le mène à saturation : illustration merveilleuse d'un fonctionnement instable.

D'autres modèles existent pour tenir compte de l'impédance d'entrée finie et de l'impédance de sortie non nulle de l'amplificateur opérationnel (fichier Pspice), des tensions d'alimentation finies (fichier Pspice) ainsi que du slew rate (fichier Pspice), des tensions de décalage (fichier Pspice) et des courants de polarisation (fichier Pspice).

En utilisant ces modèles, les étudiants simulent un certain nombre de circuits de base correspondant à des blocs fonctionnels (figure 13). Ils sont également invités à étudier les interconnexions entre ceux-ci et à se rendre compte que si on ne prend pas garde aux effets de charge, les signaux peuvent être altérés. Le problème se pose notamment lorsqu'un module RC dimensionné hors du circuit est connecté à un montage ayant une impédance d'entrée faible : le pôle associé à la cellule RC se déplace lors de l'insertion de ce module dans le circuit. 


\section{Quelques montages à}

- à simuler sur "spice" avec un modèle simplifié d'AO

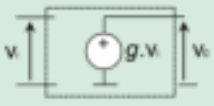

- à analyser
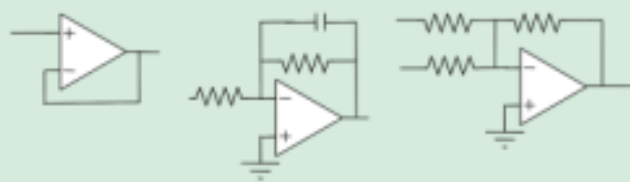

Tirez-en des idées pour votre électronique de traitement du signal.

Fig. 13 - Synthèse de la démarche

\subsubsection{Chaînes de traitement}

Dans cette section, nous présentons les chaînes de traitement proposées par les étudiants. Le rôle global de cette chaîne de traitement est d'exploiter deux tensions alternatives pour fournir une tension continue, dont l'amplitude soit proportionnelle à l'écart du capteur par rapport au fil, pour de petits déplacements du capteur, et dont le signe indique le sens de cet écart.

Pour chacune des configurations de bobines décrites dans la section 4.2.2., nous présentons une chaîne de traitement sous forme de boîtes noires. Il faudra ensuite implémenter chacun de ces blocs à l'aide de composants électroniques. Aux différents points du circuit, l'allure du signal est esquissée, permettant de vérifier la fonction réalisée par chacune des cellules construites autour de l'amplificateur.

- Configurations conf_a et conf_b

La procédure de traitement majoritairement choisie est la suivante : isolation pour s'affranchir d'un effet de charge éventuel sur les bobines, redressement simple alternance du signal de chaque bobine, extraction de la valeur moyenne par filtrage passe-bas et soustraction (figure 14). 


\section{Solution à 2 bobines de mesures}

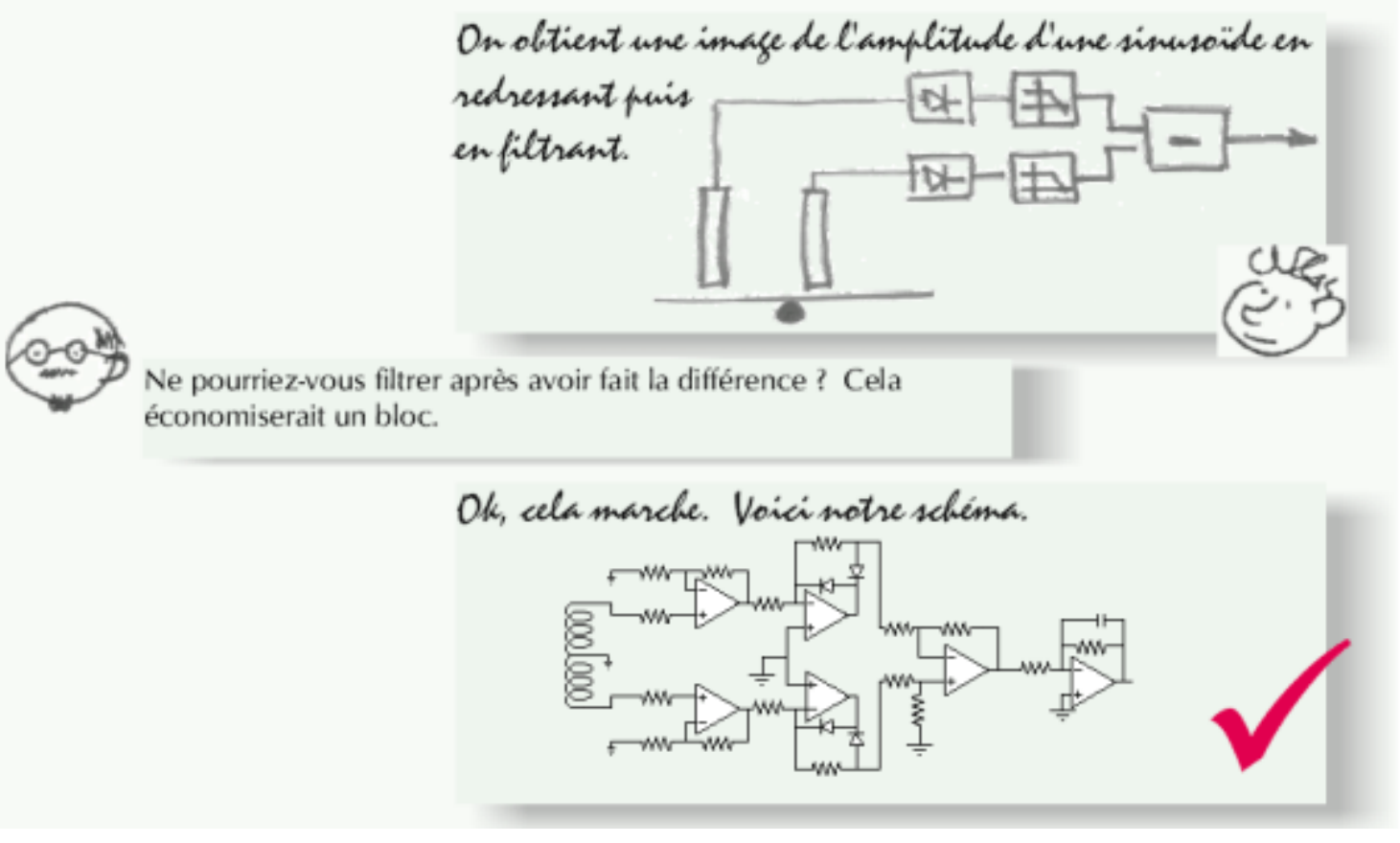

Fig. 14 - Synthèse de la démarche

- Configuration conf_c

La procédure de traitement choisie est le redressement synchrone : on redresse le signal de la bobine d'axe vertical en fonction du signal de la bobine d'axe horizontal (figure 15). Pour ce faire, on utilise un interrupteur à trois bornes : une commande, une entrée et une sortie. Si le signe du signal de commande est positif, l'élément joue le rôle de suiveur $\left(\mathrm{v}_{\mathrm{o}}=\mathrm{v}_{\mathrm{in}}\right)$, alors que dans le cas contraire, la tension de sortie est imposée à zéro. Le signe de la valeur moyenne de la tension redressée est égal au signe du cosinus du déphasage entre les signaux des deux bobines. On supposera sur base de ce signe que les tensions des bobines sont en phase ou en opposition de phase. Ce signe nous donne ainsi une information sur le côté duquel se trouve le capteur par rapport au fil. La distance entre les bobines et le fil est donnée par l'amplitude de la tension induite dans la bobine d'axe vertical. Cette méthode est plus directe et bien moins gourmande en composants électroniques que les configurations exposées précédemment, pour un même signal en fin de chaîne de mesure. 


\section{Solution à 1 bobine de mesure et 1 bobine de synchronisation}

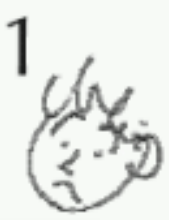

On ne sait par comment faire. On va utiliser an
montage a deuse bobines de mesure comme les autres.

Il existe des "switches" électroniques dont on peut piloter l'état via une tension. Cela permet de faire du redressement synchrone.

\section{OK?}

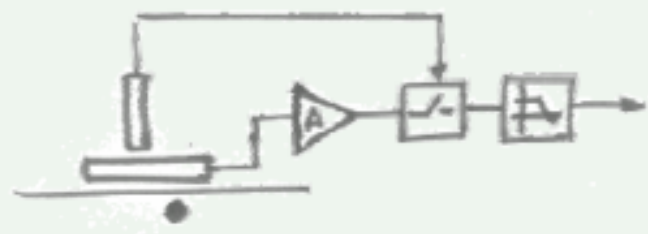

Il faut mettre en forme le signal de commande du switch..

Voici notre selima.

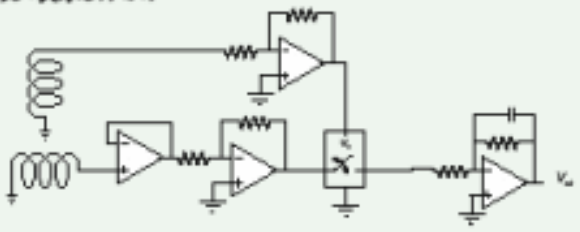

Fig. 15 - Synthèse de la démarche

\section{QUELQUES VARIANTES POSSIBLES}

On peut envisager des variantes au projet précédent.

\subsection{CAPTEUR SUR NOYAU DE FER}

Une alternative consiste à placer les deux bobines sur un noyau magnétique de ferrite en forme de E.

Dans ce cas, les bobines ont la configuration géométrique conf_a mais le calcul analytique de la tension induite par le fil n’est quantitativement plus aussi précis étant donné la canalisation du flux que provoque le noyau. Le flux intercepté par les bobines est plus important que dans le cas sans fer. De plus, ce type de capteur est moins susceptible d'être perturbé par d'autres sources de champs magnétiques ou par la présence d'éléments métalliques voisins du système.

Si la présence du noyau rend quasi impossible une étude analytique précise, on observe toutefois, expérimentalement, une allure générale de la tension en fonction de la position 
assez semblable au cas traité précédemment avec, en particulier, une plage de variation linéaire de tension à proximité du fil (figure 16).

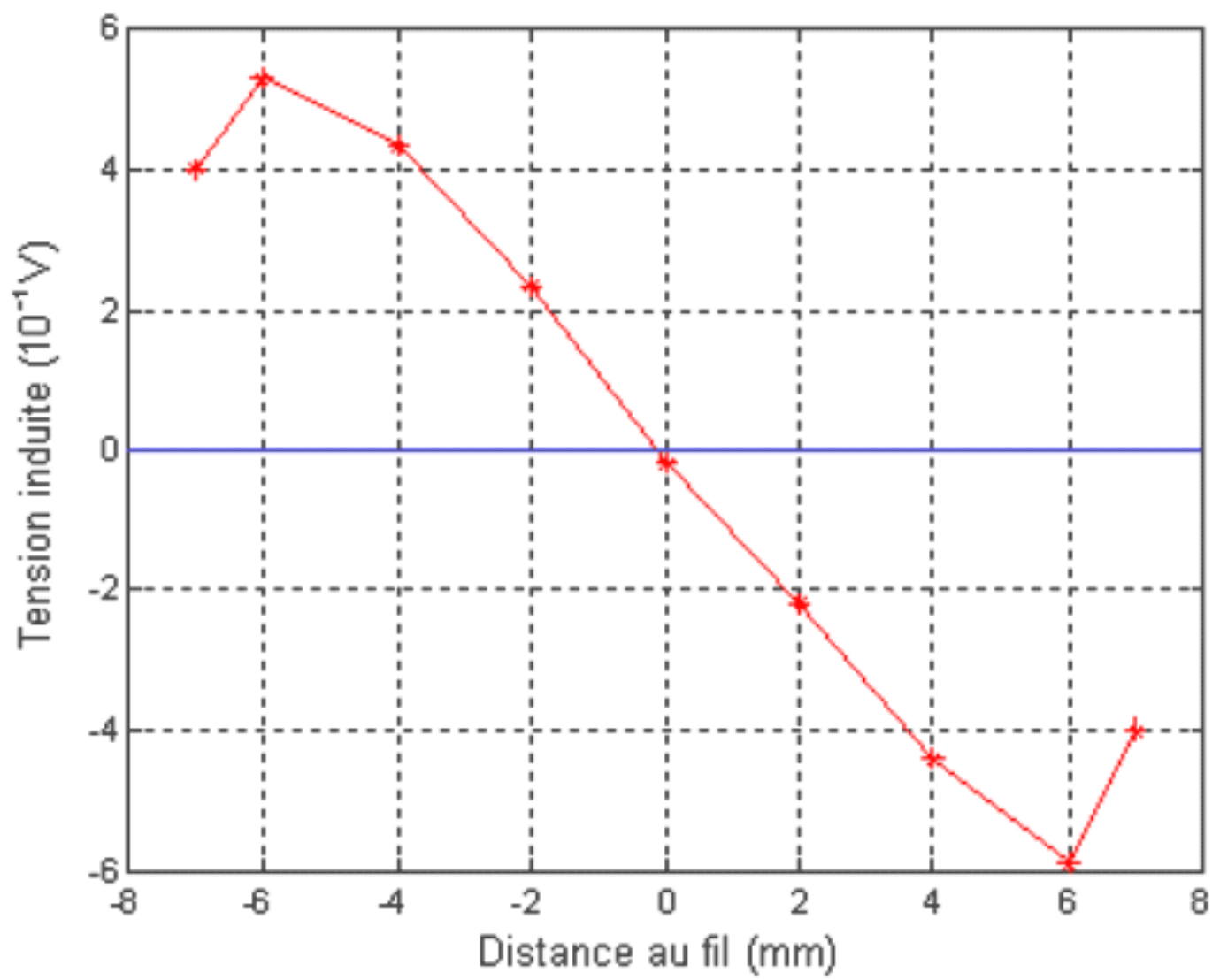

Fig. 16 - Mesure expérimentale de la réponse du capteur monté sur noyau de ferrite

On peut se contenter de cette analyse expérimentale, ou au contraire aborder par ce biais des notions plus avancées, telles que l'étude du circuit magnétique équivalent et l'introduction des notions de réluctances, ou le calcul de champs par éléments finis. Une telle démarche a été expérimentée à l’UCL pour un autre système électromagnétique [4].

\subsection{CAPTEUR OPTIQUE}

Une autre variante permet une concentration exclusive sur la partie électronique. Elle consiste à remplacer les capteurs électromagnétiques par des capteurs optiques. Il existe des capteurs optiques intégrés en réflexion comprenant à la fois l'émetteur et le récepteur, ce dernier fonctionnant dans une plage de longueur d'onde bien adaptée à celle de l'émetteur. De tels capteurs sont utilisés à l'UCL (par exemple le QRB1134, fonctionnant dans le proche infrarouge), dans le cadre d'autres projets (projet intégré en mécatronique [5,6]). La lumière reçue par ce type de capteur est émise par une diode émettrice LED et le capteur lui-même est constitué d'une photodiode.

Les capteurs optiques peuvent être utilisés pour faire du suivi de ligne, de la même manière que les capteurs électromagnétiques dans les configurations conf_a et conf_b. Il suffit pour ce faire de remplacer chaque bobine par un capteur optique, et le fil dans lequel circule le courant par une bande de couleur réfléchissant bien la lumière émise, placée sur un fond de couleur la réfléchissant peu (figure 17). 


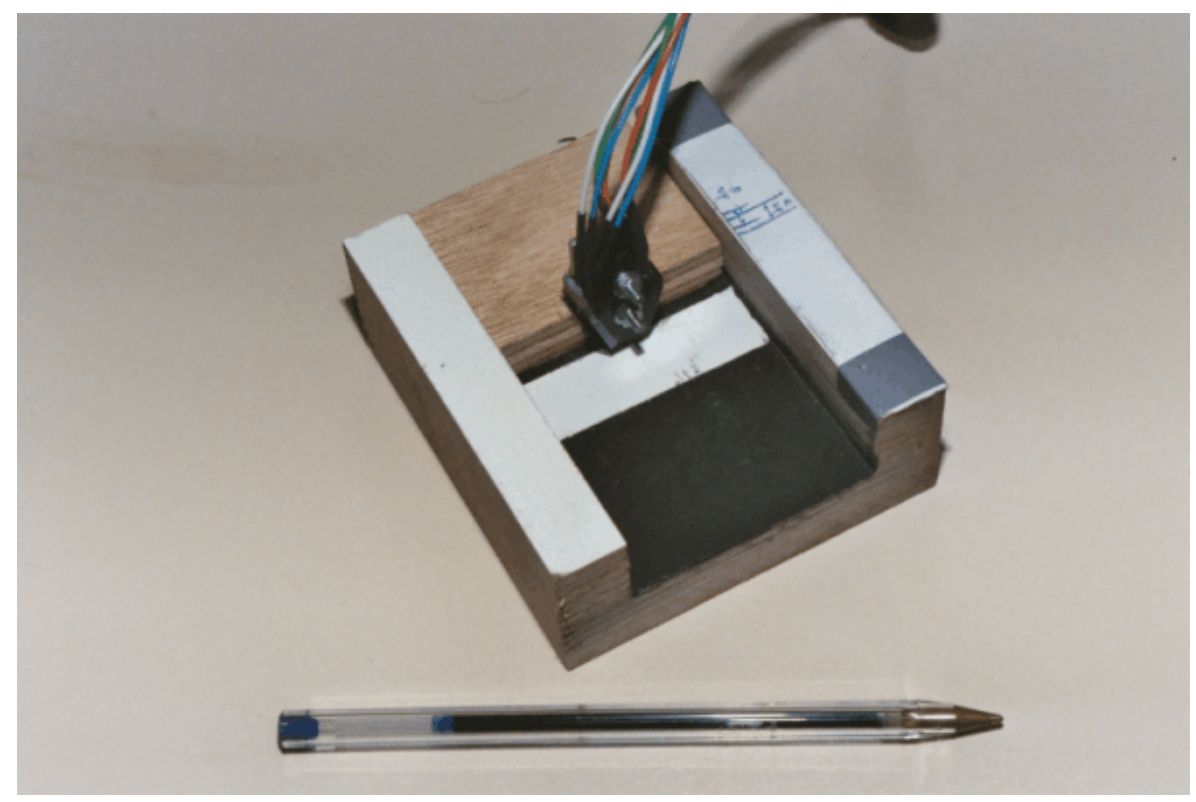

Fig. 17 - Montage des capteurs optiques

La tension de sortie du capteur peut être continue si la lumière est émise en continu. Dans ce cas, le circuit de traitement est simplifié par rapport au cas du capteur électromagnétique, les étages de redressement et de filtre pouvant être supprimés.

On aura toutefois avantage à moduler la lumière émise par la LED (figure 18). De cette manière, il est possible de s'affranchir des perturbations dues notamment à l'éclairage ambiant. Il faudra alors soit redresser et filtrer le signal du capteur, soit réaliser un filtre passebande centré autour de la fréquence de modulation. La première alternative permet d'exploiter directement et intégralement la démarche suivie dans la section IV. La seconde alternative permet d'introduire de nouvelles notions, telles que les filtres passe-haut, passe-bas, passebande et les cellules de Sallen et Key. Notons que la protection du capteur lui-même vis-à-vis de la lumière ambiante (par un confinement approprié) est également une solution intéressante.

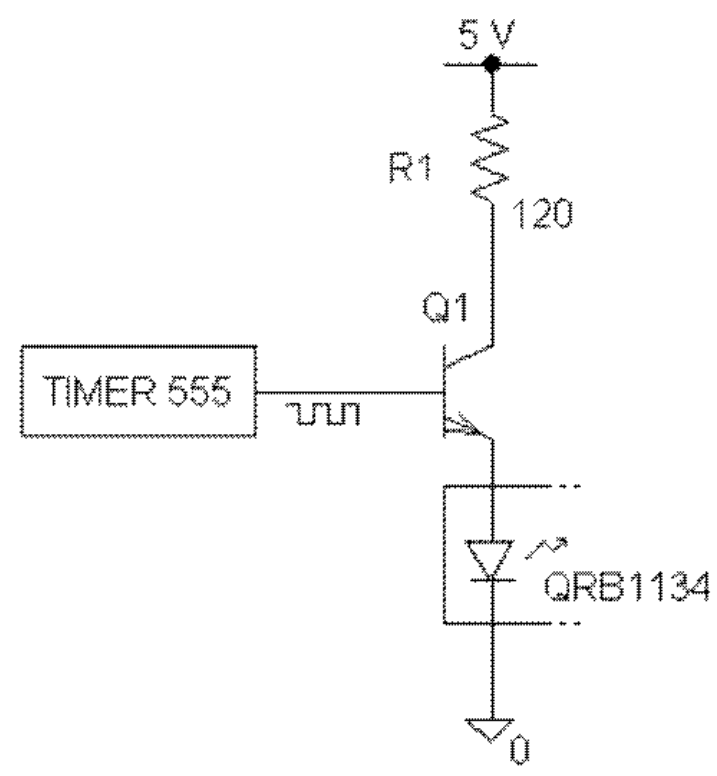

Fig. 18 - Circuit d'émetteur 


\section{CONCLUSIONS}

L'organisation matérielle de projets de l'ampleur de ceux présentés ici demande un investissement important en temps et en matériel. A l'inverse d'un cours magistral, la "surface de contact» de ces activités (produit du temps d'encadrement par le nombre d'étudiants touchés) est relativement faible pour l'équipe enseignante vu le peu d'étudiants rencontrés simultanément (consultation individuelle des groupes, étalement de l'occupation des laboratoires). L’interaction est cependant plus enrichissante pour les étudiants.

Comprendre les difficultés récurrentes des étudiants permet toutefois d'assurer un encadrement plus adéquat pour les projets suivants, en synthétisant par exemple lors d'une séance de cours l'ensemble des problèmes rencontrés par la majorité des groupes, et en esquissant les solutions de ces problèmes. De cette manière, on peut réduire grandement le temps qu’il est nécessaire de passer individuellement avec chaque groupe.

Des projets tels que ceux présentés ci-dessus permettent aux étudiants d'appliquer des notions vues de manière théorique dans certains cours préalables ou donnés en parallèle (telles que l'électromagnétisme et la théorie des circuits), ou qui seront abordées plus tard dans leur cursus (comme les imperfections des amplificateurs opérationnels).

La dynamique d'apprentissage est très différente de celle d'un cours magistral. Lors des projets, les étudiants se motivent mutuellement, que ce soit au sein des groupes ou entre ceuxci. La volonté de certains groupes d'obtenir d'excellents résultats entraîne les autres à s'investir en conséquence, afin que les niveaux de qualité ne diffèrent pas trop d'un groupe à l'autre. Loin de tout esprit de concurrence malsaine, les échanges sont nombreux entre groupes et les discussions entre les étudiants sont une aide considérable à l'avancement du projet, au même titre que les rencontres avec l'équipe enseignante lors des séances de monitorat et de laboratoire.

L'apprentissage par projet confère aux étudiants une autonomie qui se ressent dans les activités qu'ils mènent dans la suite de leurs études (et nous espérons au-delà). Nous le constatons au niveau du projet de $4^{\text {ème }}$ année, étalé sur toute l'année, où les étudiants sont amenés à concevoir un système beaucoup plus complet (système de communication ou électronique de commande d'un robot mobile[5,6]). Si l'on prévoit dans leur cursus des projets de taille et de complexité croissante, il est possible d' "amortir » la charge d'encadrement consentie pour le premier projet. On transmet de ce fait aux étudiants une compétence supplémentaire à l'issue de leurs études, qui leur donne une «valeur ajoutée » tant en vue de leur insertion dans la vie professionnelle que pour une prolongation de leur formation par la recherche.

Pour toutes ces raisons, nous sommes convaincus à l’UCL de l'efficacité de l'apprentissage par projet, si l'on dispose des moyens adéquats en matière d'encadrement des étudiants.

\section{BIBLIOGRAPHIE}

[1] E. Aguirre, C. Jacqmot, E. Milgrom, B. Raucent, A. Soucisse, Ch.Trullemans, C. Vander Borght, Devenir ingénieur par apprentissage actif

Actes du $1^{\mathrm{er}}$ Colloque de Pédagogie par projet dans l'enseignement supérieur, ENST, Brest (France), juin 2001, 7 pages 
[2] D. Grenier, P. Anciaux, Ingénieurs civils et industriels : l'enseignement supérieur de type long des sciences appliquées en Belgique francophone

Actes du CETSIS-EEA 2003, Toulouse (France), novembre 2003, pp. 193-198

[3] F. Vrins, L. De Vroey, F. Labrique, C. Trullemans, Apprentissage par projet en électricité (partie 1): Conception et réalisation d'un système de filoguidage électromagnétique

Actes du CETSIS-EEA 2003, Toulouse (France), novembre 2003, pp. 431-434

[4] L. De Vroey, D. Vanhoenacker, D. Grenier, B. Dehez, J. Gyselinck, Apprentissage par projet en électricité (partie 2) : Modélisation d'un lanceur électromagnétique Actes du CETSIS-EEA 2003, pp. 119-122

[5] D. Grenier, P. Fisette, B. Raucent, Fédérer des activités pédagogiques pour constituer un projet intégré en mécatronique: compte rendu d'innovation

Didaskalia 16, Avril 2000, pp. 163-178

[6] D. Grenier, P. Sente, P. Fisette, B. Raucent, La conception et la réalisation de robots mobiles comme éléments fédérateurs d'une formation en mécatronique

Actes du Colloque sur l'Enseignement des Technologies et des Sciences de l'Information et des Systèmes (CESTIS-EEA'99), Montpellier (France), novembre 1999, pp. 295-298. 